\title{
A New Extension of the Lomax Distribution with Properties and Applications to Failure Times Data
}

\author{
Mohamed Aboraya \\ Department of Applied Statistics and Insurance, Damietta University, Egypt. \\ mohamedaboraya17@gmail.com
}

\begin{abstract}
In this paper, we develop a new lifetime model and study some of its properties. The major justification for the practicality of theis model is based on the wider use of the Lomax model. We are also motivated to introduce the novel model since its density exhibits various important shapes such as the unimodal, right skewed and left skewed. The new model can be represented as a mixture of the exponentiated Lomax distribution. It can also be considered as a suitable model for fitting the symmetric, left skewed, right skewed and unimodal data. The maximum likelihood estimation method is used to estimate the model parameters. We show empirically the importance and flexibility of the novel model in modeling two types of aircraft windshield lifetime data sets. The proposed lifetime model is better fit than gamma Lomax, beta Lomax, exponentiated Lomax and Lomax models so the exponentiated Lomax, model is a good alternative to these models in modeling aircraft windshield data.
\end{abstract}

Keywords: Lomax model; Order Statistics; Maximum Likelihood Estimation; Quantile function; Simulation; Generating Function; Moments.

\section{Introduction}

A random variable (rv) $Z$ has the exponentiated Lx (ELx) distribution with three parameters $\alpha$ (power parameter), $\lambda$ and $\beta$ if it has cumulative distribution function (CDF) (for $z>0$ ) given by

$$
\Pi_{\alpha, \lambda, \beta}(z)=\left[1-\left(z \beta^{-1}+1\right)^{-\lambda}\right]^{\alpha} .
$$

Where $\alpha>0, \lambda>0$ and $\beta>0$ are the shape and scale parameters, respectively. Then the corresponding PDF of (1) is

$$
\pi_{\alpha, \lambda, \beta}(z)=\alpha \lambda \beta^{-1}\left(z \beta^{-1}+1\right)^{-(1+\lambda)}\left[1-\left(z \beta^{-1}+1\right)^{-\lambda}\right]^{\alpha-1},
$$

when $\alpha=1$ we get the Lomax (Lx) or the Pareto type II model with

$$
G_{\lambda, \beta}(z)=1-\left(z \beta^{-1}+1\right)^{-\lambda},
$$

and

$$
g_{\lambda, \beta}(z)=\lambda \beta^{-1}\left(z \beta^{-1}+1\right)^{-(1+\lambda)} .
$$

The Lx model was originally pioneered for modeling business failure data by Lomax (1954). The Lx distribution has found a wide application in many fields such as biological sciences, ctuarial science, engineering, size of cities, income and wealth inequality, amedical and reliability modeling. It has been applied to model data obtained from income and wealth by Harris (1968) and Atkinson and Harrison (1978), firm size by Corbellini et al. (2007), reliability and life testing by Hassan Al-Ghamdi (2009), for modeling gauge lengths data by Afify et al. (2015), for modeling bladder cancer patients data and remission times data by Yousof et al. (2016), Yousof et al. (2018) and Yousof et al. (2019) for some new properties. The Lx distribution has been also used by Adel Rastkhiz et al. (2019) for modelling entrepreneurial opportunities. Some new useful Lx extensions are recentely developd by Elsayed and Yousof (2019) and Gad et al. (2019). 
Following Yousof et al. (2016), the CDF of the Burr X generator (BrX-G) is

$$
\begin{gathered}
F_{\theta, \boldsymbol{\varphi}}(x)=2 \theta \int_{0}^{\frac{G_{\boldsymbol{\varphi}}(x)}{\bar{G}_{\boldsymbol{\varphi}}(x)}} t \exp \left(-t^{2}\right)\left[1-\exp \left(-t^{2}\right)\right]^{\theta-1} d t \\
=\left\{1-\exp \left[-\left(\frac{G_{\boldsymbol{\varphi}}(x)}{\bar{G}_{\boldsymbol{\varphi}}(x)}\right)^{2}\right]\right\}^{\theta} .
\end{gathered}
$$

The PDF of the BrX-G is given by

$$
f_{\theta, \boldsymbol{\varphi}}(x)=\frac{2 \theta g_{\varphi}(x) G_{\varphi}(x)}{\bar{G}_{\boldsymbol{\varphi}}(x)^{3}}\left\{1-\exp \left[-\left(\frac{G_{\boldsymbol{\varphi}}(x)}{\bar{G}_{\boldsymbol{\varphi}}(x)}\right)^{2}\right]\right\}^{\theta-1} \exp \left[-\left(\frac{G_{\boldsymbol{\varphi}}(x)}{\bar{G}_{\boldsymbol{\varphi}}(x)}\right)^{2}\right],
$$

where $\theta$ is the shape parameter, $g_{\varphi}(x)$ and $G_{\varphi}(x)$ denote the density and cumulative functions of the baseline model with parameter vector $\boldsymbol{\varphi}$ and $\bar{G}_{\boldsymbol{\varphi}}(x)=1-G_{\boldsymbol{\varphi}}(x)$. Inserting (1) in to (3), we get the the CDF of the Burr X ELx (BrXELx) as

$$
F_{\theta, \alpha, \lambda, \beta}(x)=\left[1-\exp \left(-\left\{\frac{\left[1-\left(x \beta^{-1}+1\right)^{-\lambda}\right]^{\alpha}}{1-\left[1-\left(x \beta^{-1}+1\right)^{-\lambda}\right]^{\alpha}}\right\}^{2}\right)\right]^{\theta} \text {. }
$$

The PDF of the BrXELx is given by

$$
\begin{aligned}
f_{\theta, \alpha, \lambda, \beta}(x)= & 2 \theta \alpha \lambda \beta^{-1}\left(x \beta^{-1}+1\right)^{-(1+\lambda)} \frac{\left[1-\left(x \beta^{-1}+1\right)^{-\lambda}\right]^{2 \alpha-1}}{\left\{1-\left[1-\left(x \beta^{-1}+1\right)^{-\lambda}\right]^{\alpha}\right\}^{3}} \\
& \times \exp \left(-\left\{\frac{\left[1-\left(x \beta^{-1}+1\right)^{-\lambda}\right]^{\alpha}}{1-\left[1-\left(x \beta^{-1}+1\right)^{-\lambda}\right]^{\alpha}}\right\}^{2}\right) \\
\times & {\left[1-\exp \left(-\left\{\frac{\left[1-\left(x \beta^{-1}+1\right)^{-\lambda}\right]^{\alpha}}{1-\left[1-\left(x \beta^{-1}+1\right)^{-\lambda}\right]^{\alpha}}\right\}^{2}\right)\right]^{\theta-1}, }
\end{aligned}
$$

when $\alpha=1$ we get $\operatorname{BrXLx}$ and when when $\alpha=\theta=1$ we get a new two parametetrs BrXLx. A rv $X$ has the BrXELx distribution if it has the CDF and PDF as (5) and (6). The reliability function $(\mathrm{RF})(R(x))$, hazard rate function $(\mathrm{HRF})(h(x))$, reversed hazard rate function (RHRF) $(r(x))$ and cumulative hazard rate function (CHRF) $(H(x))$ of $X$ are given, respectively, by

$$
\begin{aligned}
R_{\theta, \alpha, \lambda, \beta}(x) & =1-\left[1-\exp \left(-\left\{\frac{\left[1-\left(x \beta^{-1}+1\right)^{-\lambda}\right]^{\alpha}}{1-\left[1-\left(x \beta^{-1}+1\right)^{-\lambda}\right]^{\alpha}}\right\}^{2}\right)\right]^{\theta}, \\
h_{\theta, \alpha, \lambda, \beta}(x) & =2 \theta \alpha \lambda \beta^{-1} \frac{\left(x \beta^{-1}+1\right)^{-(1+\lambda)}\left[1-\left(x \beta^{-1}+1\right)^{-\lambda}\right]^{2 \alpha-1}}{\left\{1-\left[1-\left(x \beta^{-1}+1\right)^{-\lambda}\right]^{\alpha}\right\}^{3}} \\
\times & \frac{\exp \left(-\left\{\frac{\left[1-\left(x \beta^{-1}+1\right)^{-\lambda}\right]^{\alpha}}{1-\left[1-\left(x \beta^{-1}+1\right)^{-\lambda}\right]^{\alpha}}\right\}^{2}\right)}{\left[1-\exp \left(-\left\{\left[\frac{\left[1-\left(x \beta^{-1}+1\right)^{-\lambda}\right]^{\alpha}}{1-\left[1-\left(x \beta^{-1}+1\right)^{-\lambda}\right]^{\alpha}}\right]\right\}^{2}\right)\right]^{-(\theta-1)}}
\end{aligned}
$$




$$
\begin{aligned}
\times\{1- & {\left.\left[1-\exp \left(-\left\{\frac{\left[1-\left(x \beta^{-1}+1\right)^{-\lambda}\right]^{\alpha}}{1-\left[1-\left(x \beta^{-1}+1\right)^{-\lambda}\right]^{\alpha}}\right\}^{2}\right)\right]^{\theta}\right\}^{-1} } \\
r_{\theta, \alpha, \lambda, \beta}(x)= & 2 \theta \alpha \lambda \beta^{-1}\left(x \beta^{-1}+1\right)^{-(1+\lambda)} \frac{\left[1-\left(x \beta^{-1}+1\right)^{-\lambda}\right]^{2 \alpha-1}}{\left\{1-\left[1-\left(x \beta^{-1}+1\right)^{-\lambda}\right]^{\alpha}\right\}^{3}} \\
& \times \frac{\exp \left(-\left\{\frac{\left[1-\left(x \beta^{-1}+1\right)^{-\lambda}\right]^{\alpha}}{1-\left[1-\left(x \beta^{-1}+1\right)^{-\lambda}\right]^{\alpha}}\right\}^{2}\right)}{\left[1-\exp \left(-\left\{\frac{\left[1-\left(x \beta^{-1}+1\right)^{-\lambda}\right]^{\alpha}}{1-\left[1-\left(x \beta^{-1}+1\right)^{-\lambda}\right]^{\alpha}}\right\}^{2}\right)\right]^{-1}}
\end{aligned}
$$

and

$$
H_{\theta, \alpha, \lambda, \beta}(x)=-\log \left\{1-\left[1-\exp \left(-\left\{\frac{\left[1-\left(x \beta^{-1}+1\right)^{-\lambda}\right]^{\alpha}}{1-\left[1-\left(x \beta^{-1}+1\right)^{-\lambda}\right]^{\alpha}}\right\}^{2}\right)\right]^{\theta}\right\}
$$

\section{Linear representation and justification}

Consider the power series holds

$$
(1-s)^{\zeta-1}=\left.\sum_{\tau=0}^{\infty} \frac{(-s)^{\tau} \Gamma(\zeta)}{\tau ! \Gamma(\zeta-\tau)}\right|_{(|s|<1 \text { and } \zeta>0 \text { isarealnon-integer })}
$$

Applying (7) to the last term in (6) gives

$$
\begin{aligned}
& f_{\theta, \alpha, \lambda, \beta}(x)=2 \theta \alpha \lambda \beta^{-1} \sum_{i=0}^{\infty} \frac{(-1)^{i} \Gamma(\theta)}{i ! \Gamma(\theta-i)} \\
& \times \frac{\left(x \beta^{-1}+1\right)^{-(1+\lambda)}\left[1-\left(x \beta^{-1}+1\right)^{-\lambda}\right]^{2 \alpha-1}}{\left\{1-\left[1-\left(x \beta^{-1}+1\right)^{-\lambda}\right]^{\alpha}\right\}^{3}} \\
& \times \exp \left(-(1+i)\left\{\frac{\left[1-\left(x \beta^{-1}+1\right)^{-\lambda}\right]^{\alpha}}{1-\left[1-\left(x \beta^{-1}+1\right)^{-\lambda}\right]^{\alpha}}\right\}^{2}\right) \text {. }
\end{aligned}
$$

Applying the power series to the term $A$, Equation (8) becomes

$$
f_{\theta, \alpha, \lambda, \beta}(x)=2 \theta \sum_{i, j=0}^{\infty} \frac{(-1)^{i+j}(i+1)^{j} \Gamma(\theta)}{i ! j ! \Gamma(\theta-i)} \underbrace{\frac{\left\{\left[1-\left(x \beta^{-1}+1\right)^{-\lambda}\right]^{\alpha}\right\}^{2 j+1}}{\left\{1-\left[1-\left(x \beta^{-1}+1\right)^{-\lambda}\right]^{\alpha}\right\}^{2 j+3}}}_{B}
$$

Consider the series expansion

$$
(1-s)^{-\phi}=\left.\sum_{k=0}^{\infty} \frac{s^{k} \Gamma(\phi+k)}{k ! \Gamma(\phi)}\right|_{(|s|<1, \phi>0)} .
$$

Applying the expansion in (10) to (9) for the term $B$, Equation (9) becomes 
where

$$
f_{\theta, \alpha, \lambda, \beta}(x)=\sum_{j, k=0}^{\infty} v_{j, k} \pi_{[1+(1+k+2 j) \alpha], \lambda, \beta}(x),
$$

and

$$
v_{j, k}=\frac{2 \theta(-1)^{j} \Gamma(\theta) \Gamma(2 j+k+3)}{j ! k ! \Gamma(2 j+3)[1+(1+k+2 j) \alpha]} \sum_{i=0}^{\infty} \frac{(-1)^{i}(i+1)^{j}}{i ! \Gamma(\theta-i)}
$$

$$
\begin{gathered}
\pi_{[1+(1+k+2 j) \alpha], \lambda, \beta}(x)=[1+(1+k+2 j) \alpha] \\
\times \underbrace{\left[1-\left(x \beta^{-1}+1\right)^{-\lambda}\right]^{(2 j+k+1) \alpha}}_{\Pi_{[(1+k+2 j) \alpha], \lambda, \beta}(x)} \\
\times \underbrace{\alpha \lambda \beta^{-1}\left(x \beta^{-1}+1\right)^{-(1+\lambda)}\left[1-\left(x \beta^{-1}+1\right)^{-\lambda}\right]^{\alpha-1}}_{\pi_{\alpha, \lambda, \beta}(x)} .
\end{gathered}
$$

Equation (9) reveals that the density of $X$ can be expressed as a linear mixture of ELx densities. So, several mathematical properties of the new moel can be obtained by knowing those of the ELx distribution. Similarly, the cdf of the BrXELx model can also be expressed as a mixture of ELx cdfs given by

$$
F_{\theta, \alpha, \lambda, \beta}(x)=\sum_{j, k=0}^{\infty} v_{j, k} \Pi_{[1+(1+k+2 j) \alpha], \lambda, \beta}(x)
$$

where $\Pi_{[1+(1+k+2 j) \alpha], \lambda, \beta}(x)$ is the cdf of the ELx model with power parameter $[1+$ $(1+k+2 j) \alpha]$.

The major justification for the practicality of the new BrXELx model is based on the wider use of the Lx model. We are also motivated to introduce the BrXELx model since its density exhibits various important shapes such as the unimodal, the right skewed and the left skewed (see figure 1). The new model can be viewed as a mixture of the exponentiated Lomax distributions (see Subsection 2.1). It can also be considered as a suitable model for fitting the symmetric, left skewed, right skewed, and unimodal data sets (see aplications Section). The maximum likelihood estimation method is used to estimate the BrXELx parameters. We show empirically the importance and flexibility of the new Lx model in modeling two types of aircraft windshield lifetime data sets. The BrXELx model is better fit than some other competitive models so the BrXELx model is a good alternative to in modeling failure and service times data. 

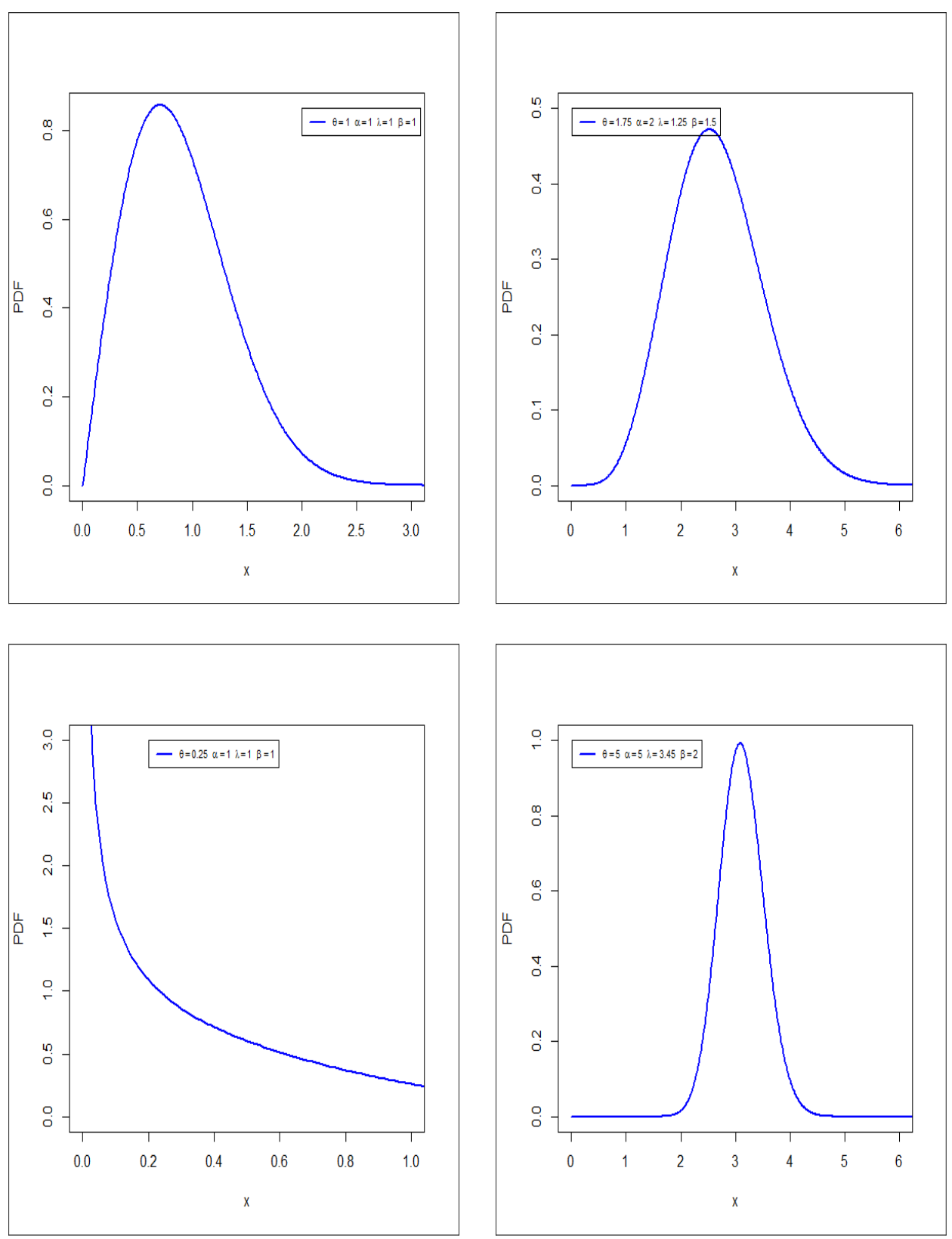

Figure 1: Plots of the BrXELx PDF. 

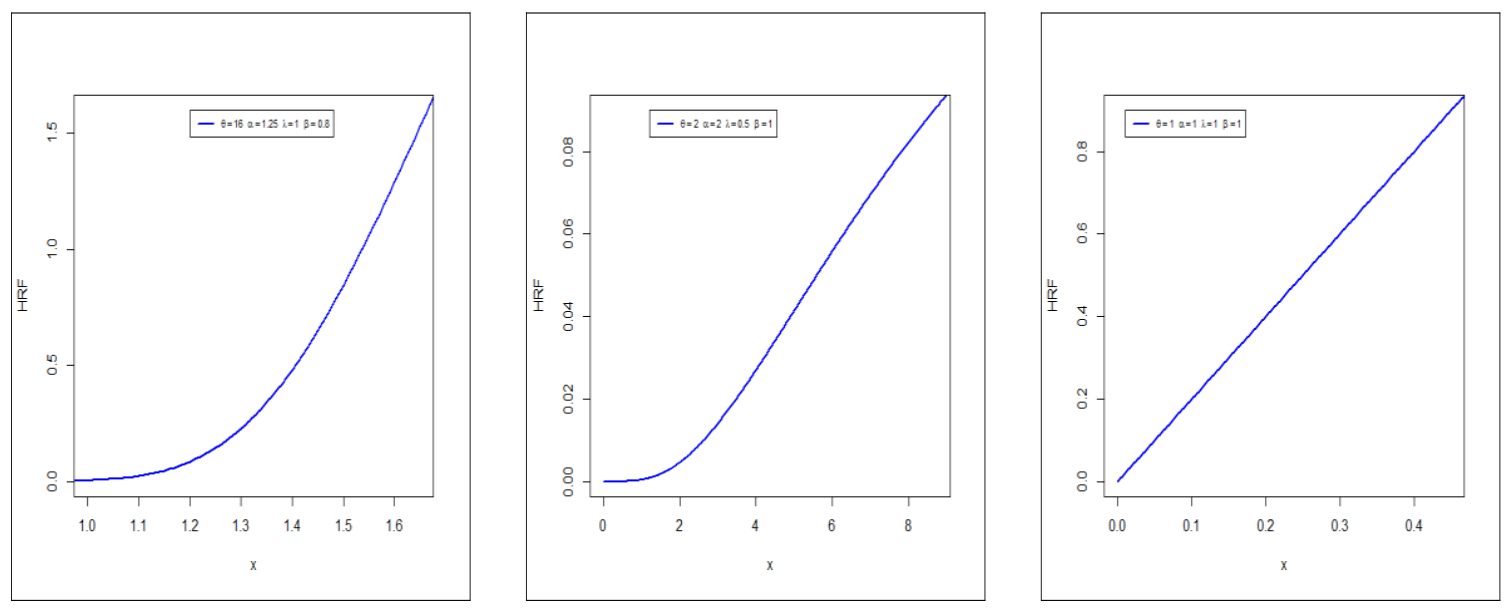

Figure 2: Plots of the BrXELx HRF.

\section{Mathematical and statistical properties \\ 3.1 Moments and generating function}

The $r^{\text {th }}$ ordinary moment of $X$ is given by

$$
\mu_{r}^{\prime}=\mathbf{E} X^{r}=\int_{-\infty}^{\infty} x^{r} f(x) d x
$$

Then we obtain

$$
\mu_{r}^{\prime}=\left.\sum_{j, k=0}^{\infty} \sum_{w=0}^{r} \frac{v_{j, k} \beta^{r}(-1)^{w}}{[1+(1+k+2 j) \alpha]^{-1}}\left(\begin{array}{l}
r \\
w
\end{array}\right) \mathbf{B}\left([1+(1+k+2 j) \alpha], 1+\frac{w-r}{\lambda}\right)\right|_{(\lambda>r)},
$$

where

$$
\mathbf{B}\left(a_{1} ; a_{2}\right)=\int_{0}^{1} z^{a_{1}-1}(1-z)^{a_{2}-1} d z,
$$

is the complete beta function. Setting $r=1$ in $\mu_{r}^{\prime}$, we have the mean of $X$

$$
\begin{gathered}
\mathbf{E} X=\mu_{1}^{\prime}=\sum_{j, k=0}^{\infty} \sum_{w=0}^{1} \frac{v_{j, k} \beta(-1)^{w}}{[1+(1+k+2 j) \alpha]^{-1}}\left(\begin{array}{l}
1 \\
w
\end{array}\right) \\
\times\left.\mathbf{B}\left([1+(1+k+2 j) \alpha], 1+\frac{w-1}{\lambda}\right)\right|_{(\lambda>1)} .
\end{gathered}
$$

Setting $r=2.3$ and 4 in (13), we have the $2^{n d}, 3^{r d}$ and the $4^{t h}$ moments about the origin

$$
\begin{aligned}
\mathbf{E} X^{2}=\mu_{2}^{\prime}= & \sum_{j, k=0}^{\infty} \sum_{w=0}^{2} \frac{v_{j, k} \beta^{2}(-1)^{w}}{[1+(1+k+2 j) \alpha]^{-1}}\left(\begin{array}{l}
2 \\
w
\end{array}\right) \\
& \times\left.\mathbf{B}\left([1+(1+k+2 j) \alpha], 1+\frac{w-2}{\lambda}\right)\right|_{(\lambda>2)},
\end{aligned}
$$


and

$$
\begin{aligned}
\mathbf{E} X^{3}=\mu_{3}^{\prime}= & \sum_{j, k=0}^{\infty} \sum_{w=0}^{3} \frac{v_{j, k} \beta^{3}(-1)^{w}}{[1+(1+k+2 j) \alpha]^{-1}}\left(\begin{array}{l}
3 \\
w
\end{array}\right) \\
& \times\left.\mathbf{B}\left([1+(1+k+2 j) \alpha], 1+\frac{w-3}{\lambda}\right)\right|_{(\lambda>3)},
\end{aligned}
$$

$$
\begin{aligned}
\mathbf{E} X^{4}=\mu_{4}^{\prime}= & \sum_{j, k=0}^{\infty} \sum_{w=0}^{4} \frac{v_{j, k} \beta^{4}(-1)^{w}}{[1+(1+k+2 j) \alpha]^{-1}}\left(\begin{array}{l}
4 \\
w
\end{array}\right) \\
& \times\left.\mathbf{B}\left([1+(1+k+2 j) \alpha], 1+\frac{w-4}{\lambda}\right)\right|_{(\lambda>4)},
\end{aligned}
$$

which can be used to obtain the central moments. The moment generating function (MGF) $M_{X}(t)=\mathbf{E}\left(e^{t X}\right)$ of $X$. Clearly, the first one can be derived from equation (10) as

$$
\begin{aligned}
& M_{X}(t)=\sum_{j, k=0}^{\infty} \sum_{w=0}^{r} \frac{v_{j, k} t^{r} \beta^{r}(-1)^{w}}{[1+(1+k+2 j) \alpha] r !}\left(\begin{array}{l}
r \\
w
\end{array}\right) \\
& \times\left.\mathbf{B}\left([1+(1+k+2 j) \alpha], 1+\frac{w-r}{\lambda}\right)\right|_{(\lambda>r)},
\end{aligned}
$$

\subsection{Incomplete moments and mean deviations}

The $s^{\text {th }}$ incomplete moment, say $\mathbf{I}_{s}(t)$, of $X$ can be expressed from (10) as

$$
\begin{aligned}
\mathbf{I}_{s}(t)= & \int_{-\infty}^{t} x^{s} f(x) d x \\
& =\sum_{j, k=0}^{\infty} \sum_{w=0}^{s} \frac{v_{j, k} \beta^{s}(-1)^{w}}{[1+(1+k+2 j) \alpha]^{-1}}\left(\begin{array}{l}
s \\
w
\end{array}\right) \\
& \times\left.\mathbf{B}_{t}\left([1+(1+k+2 j) \alpha], 1+\frac{w-s}{\lambda}\right)\right|_{(\lambda>s)},
\end{aligned}
$$

where

$$
\mathbf{B}_{y}\left(a_{1} ; a_{2}\right)=\int_{0}^{y} z^{a_{1}-1}(1-z)^{a_{2}-1} d z,
$$

is the incomplete beta function. The mean deviations about the mean

and about the median

$$
\mathbf{M D}_{(\mathbf{E} X)}=\mathbf{E}(|X-\mathbf{E}(X)|)=2 \mathbf{E} X F(\mathbf{E} X)-2 \mathbf{I}_{1} \mathbf{E} X,
$$

$$
\mathbf{M D}_{(\operatorname{Median}(X))}=\mathbf{E}(|X-\operatorname{Median}(X)|)=\mathbf{E} X-2 \mathbf{I}_{1}(\operatorname{Median}(X)),
$$

where $F(\mathbf{E} X)$ is easily calculated from $(5)$ and $I_{1}(t)$ is the first incomplete moment given by $\mathbf{I}_{s}(t)$ with $s=1$. Now, we provide two ways to determine $M D_{(\mathbf{E} X)}$ and $M D_{(\operatorname{Median}(X))}$. The $\mathbf{I}_{1}(t)$ can be derived from (14) as

$$
\begin{aligned}
& \mathbf{I}_{1}(t)=\sum_{j, k=0}^{\infty} \sum_{w=0}^{s} \frac{v_{j, k} \beta(-1)^{w}}{[1+(1+k+2 j) \alpha]^{-1}}\left(\begin{array}{l}
1 \\
w
\end{array}\right) \\
& \times\left.\mathbf{B}_{t}\left([1+(1+k+2 j) \alpha], 1+\frac{w-1}{\lambda}\right)\right|_{(\lambda>1)} .
\end{aligned}
$$




\subsection{Probability weighted moments}

The probability weighted moment (PWMs) are expectations of certain functions of a random variable and they can be defined for any random variable whose ordinary moments exist. The PWM method can generally be used for estimating parameters of a distribution whose inverse form cannot be expressed explicitly. defined by

The $(s, r)^{t h}$ PWM of $X$ following the BrXELx model, say $\rho_{s, r}$, is formally

$$
\rho_{s, r}=\mathbf{E}\left\{X^{s} F_{\theta, \lambda, \beta}(x)^{r}\right\}=\int_{-\infty}^{\infty} x^{s} F_{\theta, \alpha, \lambda, \beta}(x)^{r} f_{\theta, \lambda, \beta}(x) d x .
$$

Using (5), (6) we can write

where

$$
F_{\theta, \alpha, \lambda, \beta}(x)^{r} f_{\theta, \alpha, \lambda, \beta}(x)=\sum_{j, k=0}^{\infty} w_{j, k} \pi_{[1+(1+k+2 j) \alpha], \lambda, \beta}(x),
$$

$$
w_{j, k}=\frac{2 \theta(-1)^{j} \Gamma(2 j+k+3)}{j ! k ! \Gamma(2 j+3)[1+(1+k+2 j) \alpha]} \sum_{i=0}^{\infty}(-1)^{i}(i+1)^{j}\left(\begin{array}{l}
\theta(r+1)-1 \\
i
\end{array}\right) .
$$

Then, the $(s, r)^{t h}$ PWM of $X$ can be expressed as

$$
\begin{aligned}
& \rho_{s, r}=\sum_{j, k=0}^{\infty} \sum_{w=0}^{s} \frac{w_{j, k} \beta^{s}(-1)^{w}}{[1+(1+k+2 j) \alpha]^{-1}}\left(\begin{array}{l}
s \\
w
\end{array}\right) \\
& \times\left.\mathbf{B}\left([1+(1+k+2 j) \alpha], 1+\frac{w-s}{\lambda}\right)\right|_{(\lambda>s)} .
\end{aligned}
$$

\subsection{Moments of the reversed residual life}

The $n^{\text {th }}$ moment of the reversed residual life, say

$$
M_{n}(t)=\left.\mathbf{E}\left[(t-X)^{n}\right]\right|_{(X \leq t, t>0, n=1,2, \ldots)}
$$

uniquely determines $F_{\theta, \lambda, \beta}(x)$. We obtain

$$
M_{n}(t)=\frac{1}{F_{\theta, \lambda, \beta}(x)} \int_{0}^{t}(t-x)^{n} d F_{\theta, \alpha, \lambda, \beta}(x) .
$$

Then, the $n^{\text {th }}$ moment of the reversed residual life of $X$ becomes

where

$$
\begin{gathered}
M_{n}(t)=F_{\theta, \alpha, \lambda, \beta}(x)^{-1} \sum_{j, k=0}^{\infty} \sum_{r=0}^{n} \sum_{w=0}^{n} v_{j, k, r, w} \\
\times\left.\mathbf{B}_{t}\left([1+(1+k+2 j) \alpha], 1+\frac{w-n}{\lambda}\right)\right|_{(\lambda>n)} .
\end{gathered}
$$

$$
v_{j, k, r, w}=v_{j, k}(-1)^{r+w} t^{n-r}[1+(1+k+2 j) \alpha] \beta^{n}\left(\begin{array}{l}
n \\
r
\end{array}\right)\left(\begin{array}{l}
n \\
w
\end{array}\right) .
$$

The mean inactivity time (MIT) or mean waiting time (MWT) also called the mean reversed residual life function, is given by

$$
M_{1}(t)=\left.\mathbf{E}[(t-X)]\right|_{X \leq t},
$$

and it represents the waiting time elapsed since the failure of an item on condition that 
this failure had occurred in $(0, t)$. The MIT of the BrXELx distribution can be obtained easily by setting $n=1$ in the above equation. Then, the $n^{\text {th }}$ moment of the residual life of $X$ comes from the $M_{n}(t)$ equation by changing $F_{\theta, \lambda, \beta}(x)$ by $1-F_{\theta, \lambda, \beta}(x)$.

\subsection{Order statistics}

Order statistics make their appearance in many areas of statistical theory and practice. Let $X_{1}, \ldots, X_{n}$ be a random sample from the BrXELx distribution and let $X_{1: n}, \ldots, X_{n: n}$ be the corresponding order statistics. The PDF of $i^{\text {th }}$ order statistic, say $X_{i: n}$, can be written as

$$
f_{\theta, \lambda, \beta}^{(i: n)}(x)=\frac{f_{\theta, \lambda, \beta}(x)}{\mathrm{B}(i, n-i+1)} \sum_{j=0}^{n-i}(-1)^{j}\left(\begin{array}{l}
n-i \\
j
\end{array}\right) F_{\theta, \lambda, \beta}(x)^{j+i-1},
$$

where $B(\cdot, \cdot)$ is the beta function. Using $(5),(6)$ and $f_{\theta, \lambda, \beta}^{(i: n)}(x)$ we get

where

$$
f_{\theta, \lambda, \beta}(x) F_{\theta, \lambda, \beta}(x)^{j+i-1}=\sum_{w, k=0}^{\infty} t_{w, k} \pi_{[1+(1+k+2 w) \alpha], \lambda, \beta}(x)
$$

$$
t_{w, k}=\frac{2 \theta(-1)^{w} \Gamma(2 w+k+3)}{w ! k ! \Gamma(2 w+3)[1+(1+k+2 w) \alpha]} \sum_{m=0}^{\infty}(-1)^{m}(m+1)^{w}\left(\begin{array}{l}
\theta(j+i)-1 \\
m
\end{array}\right) .
$$

The PDF of $X_{i: n}$ can be expressed as

$$
f_{\theta, \lambda, \beta}^{(i: n)}(x)=\sum_{w, k=0}^{\infty} \sum_{j=0}^{n-i} \frac{(-1)^{j}\left(\begin{array}{l}
n-i \\
j
\end{array}\right) t_{w, k}}{\mathrm{~B}(i, n-i+1)} \pi_{[1+(1+k+2 w) \alpha], \lambda, \beta}(x) .
$$

Then, the density function of the BrXELx order statistics is a mixture of ELx. Based on the last equation, we note that the properties of $X_{i: n}$ follow from those properties of $Y_{2 w+k+2}$. For example, the moments of $X_{i: n}$ can be expressed as

where

$$
\begin{gathered}
\mathbf{E}\left(X_{i: n}^{q}\right)=\sum_{w, k=0}^{\infty} \sum_{j=0}^{n-i} \sum_{m=0}^{q} t_{w, k, j, m} \beta^{q}\left(\begin{array}{c}
q \\
m
\end{array}\right) \\
\times\left.\mathbf{B}\left([1+(1+k+2 w) \alpha], 1+\frac{m-q}{\lambda}\right)\right|_{(\lambda>q)} .
\end{gathered}
$$

$$
t_{w, k, j, m}=\frac{t_{w, k}(-1)^{j+m} \mathrm{~B}^{-1}(i, n-i+1)}{[1+(1+k+2 w) \alpha]}\left(\begin{array}{l}
n-i \\
j
\end{array}\right)\left(\begin{array}{l}
q \\
m
\end{array}\right)
$$

\section{Parameter etimation}

Several approaches for estimating parameters were proposed in the literature but the maximum likelihood method is the most commonly employed. So, we consider the estimation of the unknown parameters of this family from complete samples only by maximum likelihood. Let $x_{1}, \ldots, x_{n}$ be a random sample from the BrXELx model with parameters $\theta$ and $\boldsymbol{\varphi}$. Let $\Theta=(\theta, \alpha, \lambda, \beta)^{\top}$ be the $4 \times 1$ parameter vector. For determining the MLE of $\Theta$, we have the log-likelihood function 
where

$$
\begin{aligned}
\mathbf{L}=\mathbf{L}(\Theta)= & n \log 2+n \log \theta+n \log \alpha+n \log \lambda-n \log \beta-(1+\lambda) \sum_{i=1}^{n} \log \left(1+x_{i} \beta^{-1}\right) \\
& -\sum_{i=1}^{n}\left(\frac{s_{i}}{1-s_{i}}\right)^{2}+(\theta-1) \sum_{i=1}^{n} \log \left\{1-\exp \left[-\left(\frac{s_{i}}{1-s_{i}}\right)^{2}\right]\right\},
\end{aligned}
$$

$$
s_{i}=\left[1-\left(1+x_{i} \beta^{-1}\right)^{-\lambda}\right]^{\alpha} .
$$

The components of the score vector, $\mathbf{U}(\Theta)=\left(\frac{\partial}{\partial \theta} \mathbf{L}(\Theta), \frac{\partial}{\partial \alpha} \mathbf{L}(\Theta), \frac{\partial}{\partial \lambda} \mathbf{L}(\Theta), \frac{\partial}{\partial \boldsymbol{\beta}} \mathbf{L}(\Theta)\right)^{\top}$, are availble if needed, Setting the nonlinear system of equations $U_{\theta}=0, U_{\alpha}=0, U_{\gamma}=0$ and $U_{\beta}=0$ and solving them simultaneously yields the MLE $\widehat{\Theta}=(\hat{\theta}, \hat{\lambda}, \hat{\beta},)^{\top}$. To solve these equations, it is usually more convenient to use nonlinear optimization methods such as the quasi-Newton algorithm to numerically maximize $\mathbf{L}(\Theta)$.

\section{Simulation studies}

We simulate the BrXELx model by taking $n=20,50,150,500$ and 1000 . For each sample size, we evaluate the ML estimations (MLEs) of the parameters using the optim function of the $\mathrm{R}$ software. Then, we repeat this process 1000 times and compute the averages of the estimates (AEs), biases (Bias) and mean squared errors (MSEs). Table 1 gives all simulation results. The values in Table 1 indicate that the MSEs and the Bias of $\hat{\theta}, \hat{\alpha}, \hat{\lambda}$ and $\hat{\beta}$ decay toward zero when $n$ increases for all settings of $\theta, \alpha, \lambda$ and $\beta$, as expected under first-under asymptotic theory. The AEs of the parameters tend to be closer to the true parameter values ( $\theta=2, \alpha=1.5, \lambda=0.6$ and $\beta=0.8$ ) when $n$ increases. This fact supports that the asymptotic normal distribution provides an adequate approximation to the finite sample distribution of the MLEs. Table 1 gives the AEs, Bias and MSEs based on 1000 simulations. 
Table 1: AEs, Bias and MSE based on 1000 simulations.

\begin{tabular}{|c|c|c|c|c|}
\hline $\mathrm{n}$ & $\Theta$ & $\mathrm{AE}$ & Bias & MSE \\
\hline \multirow[t]{4}{*}{20} & $\theta$ & 2.2099493 & 0.2099493 & 0.0207493 \\
\hline & $\alpha$ & 1.5106461 & 0.0187636 & 0.9024658 \\
\hline & $\lambda$ & 0.6003500 & 0.0003500 & 0.0009674 \\
\hline & $\beta$ & 0.8149436 & 0.0149436 & 0.0199576 \\
\hline \multirow[t]{4}{*}{50} & $\theta$ & 2.1166858 & 0.11668580 & 0.5357800 \\
\hline & $\alpha$ & 1.5092566 & 0.00925660 & 0.0094288 \\
\hline & $\lambda$ & 0.5995366 & -0.0004634 & 0.0005031 \\
\hline & $\beta$ & 0.8101070 & 0.01010700 & 0.0080147 \\
\hline \multirow[t]{4}{*}{150} & $\theta$ & 2.1475741 & 0.1475741 & 0.9024658 \\
\hline & $\alpha$ & 1.5190324 & 0.0190324 & 0.0024648 \\
\hline & $\lambda$ & 0.5959778 & -0.0040222 & 0.0002627 \\
\hline & $\beta$ & 0.8174878 & 0.0174878 & 0.0037165 \\
\hline \multirow[t]{4}{*}{500} & $\theta$ & 2.1359867 & 0.1359867 & 0.0774530 \\
\hline & $\alpha$ & 1.5197785 & 0.0197785 & 0.0016683 \\
\hline & $\lambda$ & 0.5950373 & -0.0049627 & 0.0001079 \\
\hline & $\beta$ & 0.8174235 & 0.0174235 & 0.0012894 \\
\hline \multirow[t]{4}{*}{1000} & $\theta$ & 2.1366144 & 0.0136614 & 0.0470211 \\
\hline & $\alpha$ & 1.5203488 & 0.0203488 & 0.0010398 \\
\hline & $\lambda$ & 0.5946883 & -0.0053117 & 0.0000703 \\
\hline & $\beta$ & 0.8177539 & 0.0177539 & 0.0007934 \\
\hline
\end{tabular}




\section{Applications}

In this section, we provide two applications to two real data sets to show the importance and flexibility of the BrXELx distribution. We compare the fit of the BrXELx with competitve Lx models namely: the ELx model (Gupta et al., 1998), the gamma Lomax (GaLx) model (Cordeiro et al., 2015), the beta Lomax (BLX) model (Lemonte and Cordeiro, 2013) and Lx model. The CDFs of these distributions are, respectively, given by (for $x>0$ and $\alpha, \beta, \lambda, a>0$ ):

$$
\begin{gathered}
F_{E L x}(x ; \alpha, \beta, \lambda)=\left[1-\left(1+x \beta^{-1}\right)^{-\lambda}\right]^{\alpha}, \\
F_{G a L x}(x ; \alpha, \beta, \lambda)=\Gamma^{-1}(\alpha) \Gamma\left(\alpha ; \lambda \log \left(1+x \beta^{-1}\right)\right),
\end{gathered}
$$

and

$$
F_{B L x}(x ; \alpha, \beta, \theta, \lambda)=\frac{1}{B(\alpha, \theta)} B_{\left[1-\left(1+x \beta^{-1}\right)^{-\lambda}\right]}(\alpha, \theta),
$$

where $\Gamma(\cdot)$ is the gamma function, $\Gamma(\because \cdot)$ is the incomplete gamma function.

The first real data set represents the data on failure times of 84 aircraft windshield given in Murthy et al. (2004). The data are:

$0.0400,1.866,2.3850,3.443,0.3010,1.876,2.4810,3.467,0.309,1.8990,2.610,3.4780$, $0.55700,1.9110,2.625,3.5780,0.943,1.9120,2.632,3.5950,1.0700,1.914,2.6460$, $3.699,1.1240,1.981,2.661,3.7790,1.248,2.0100,2.688,3.9240,1.2810,2.038,2.820,3$, $4.035,1.281,2.0850,2.890,4.121,1.3030,2.089,2.902,4.167,1.4320,2.097,2.934$, $4.2400,1.480,2.135,2.962,4.2550,1.505,2.154,2.9640,4.278,1.506,2.190,3.000$, $4.3050,1.56800,2.1940,3.103,4.376,1.615,2.2230,3.114,4.449,1.6190,2.224$, $3.1170,4.485,1.652,2.2290,3.166,4.570,1.652,2.3000,3.344,4.602,1.7570,2.324$, $3.3760,4.663$.

The second real data set represents the data on service times of 63 aircraft windshield given in Murthy et al. (2004). The data are:

$0.046,1.436,2.592,0.140,1.492,2.600,0.150,1.580,2.670,0.248,1.7190,2.717$, $0.2800,1.794,2.819,0.3130,1.915,2.820,0.389,1.9200,2.878,0.487,1.9630,2.950$, $0.622,1.978,3.0030,0.9000,2.053,3.1020,0.952,2.065,3.3040,0.9960,2.117,3.483$, $1.0030,2.137,3.500,1.0100,2.141,3.6220,1.085,2.163,3.6650,1.092,2.183,3.695$, $1.1520,2.2400,4.015,1.183,2.3410,4.628,1.2440,2.435,4.806,1.249,2.4640,4.881$, $1.262,2.5430,5.140$. These data sets were recently studied by Tahir et al. (2015). The unit for measurement is $1000 \mathrm{~h}$ for both data sets.

The total time test (TTT) plot is an important graphical approach to verify whether the data can be applied to a specific distribution or not (see Aarset, 1987). According to Aarset (1987), the empirical version of the TTT plot is given by plotting

$$
T(r / n)=\left[\sum_{i=1}^{r} y_{i: n}+(n-r) y_{r: n}\right]\left(\sum_{i=1}^{n} y_{i: n}\right)^{-1}
$$

against $r / n$, where $r=1, \ldots, n$ and $y_{i: n}(i=1, \ldots, n)$ are the order statistics of the sample. Aarset (1987) showed that the HRF is constant if the TTT plot is graphically presented as a straight diagonal, the HRF is increasing (or decreasing) if the TTT plot is 
concave (or convex). The HRF is $\mathbf{U}$-shaped (bathtub) if the TTT plot is firstly convex and then concave, if not, the HRF is unimodal. The TTT plots the three real data sets is presented in Figure 3. This plot indicates that the empirical HRFs of the the three data sets are decreasing, decreasing and unimodal. Figure 3 indicates that the empirical HRFs of the both data sets are decreasing.

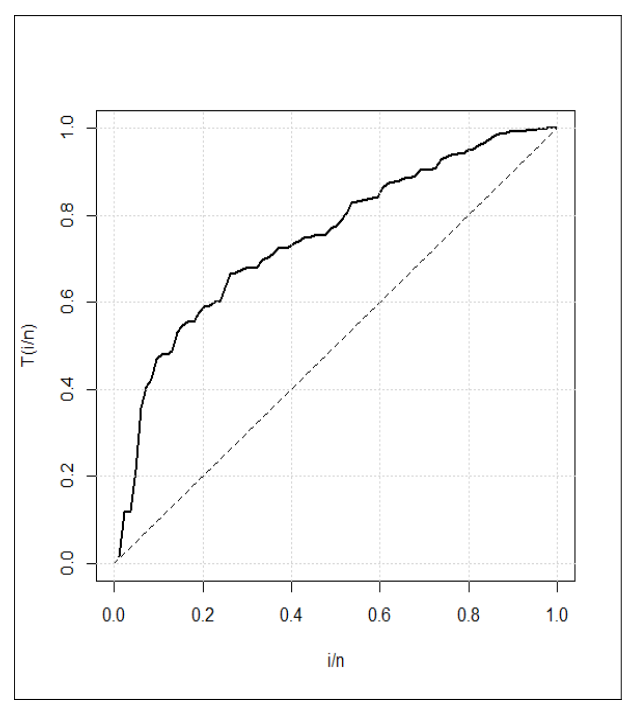

Data Set I

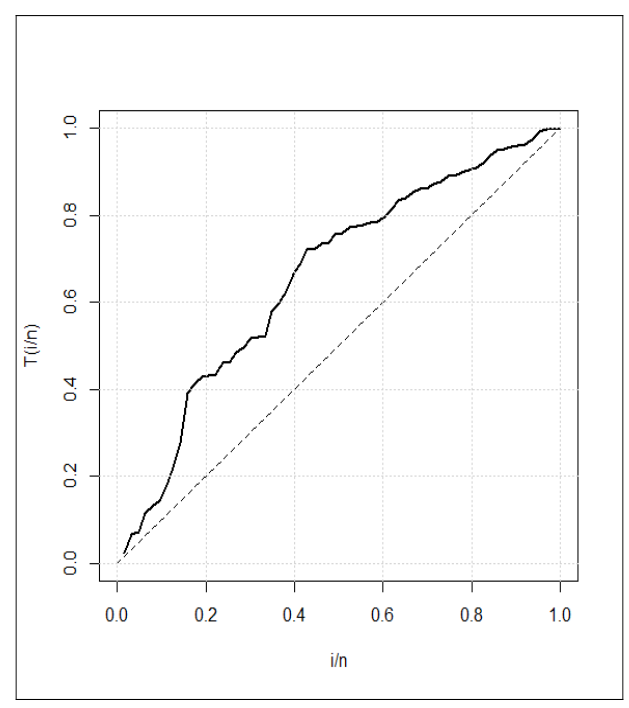

Data Set II

Figure 3: TTT plots.

In order to compare the distributions, the estimated log-likelihood values $\hat{\mathbf{L}}$, Akaike Information Criteria (AIC), Cramer von Mises $\left(W^{*}\right)$ and Anderson-Darling $\left(A^{*}\right)$ goodness of-fit statistics were calculated for all models. The statistics $W^{*}$ and $A^{*}$ are described in detail in Chen and Balakrishnan (1995). In general, it can be chosen as the best model which has the smaller values of the AIC, $W^{*}$ and $A^{*}$ statistics and the larger values of $\hat{\mathbf{L}}$. The required computations are obtained by using the "maxLik" and "goftest" sub-routines in R-software. The analysis results of both these applications are listed in Tables 2-5. MLEs, standard errors (SEs) of the estimates (in parentheses) for the two data sets are listed on Tables 2 and 4. These results show that the new distribution has the lowest $A I C, W^{*}$ and $A^{*}$ values and, has the biggest estimated $-\hat{\mathbf{L}}$ among all the fitted models. Hence, it could be chosen as the best model under these criteria. From tables 3 and 5, the proposed BrXELx lifetime model is much better (preferable) than gamma Lomax, beta Lomax, exponentiated Lomax and Lomax models so the exponentiated Lomax, model is a good alternative to these models in modeling aircraft windshield data. MLEs, standard erros (SEs) of the estimates (in parentheses) for the two data sets are lised on Tables 2 and 4. 
Table 2: MLEs and SEs for the first data set.

\begin{tabular}{|c|c|c|c|c|}
\hline Model & $\widehat{\alpha}$ & $\widehat{\beta}$ & $\widehat{\theta}$ & $\hat{\lambda}$ \\
\hline BrXELx & $\begin{array}{c}9.963 \times e^{-1} \\
(0.0001)\end{array}$ & $\begin{array}{c}3.389 \times e^{5} \\
(124.7505)\end{array}$ & $\begin{array}{r}8.347 \times e^{-1} \\
(0.0001)\end{array}$ & $\begin{array}{l}7.539 \times e^{4} \\
(0.0001)\end{array}$ \\
\hline BLx & $\begin{array}{c}3.604 \\
(0.6187)\end{array}$ & $\begin{array}{c}118.837 \\
(63.7145)\end{array}$ & $\begin{array}{l}33.639 \\
(9.238)\end{array}$ & $\begin{array}{c}4.831 \\
(429.00)\end{array}$ \\
\hline ELx & $\begin{array}{c}3.626 \\
(0.624)\end{array}$ & $\begin{array}{c}26257.680 \\
(99.742)\end{array}$ & & $\begin{array}{l}20074.509 \\
(2041.826)\end{array}$ \\
\hline GLx & $\begin{array}{c}3.588 \\
(0.513)\end{array}$ & $\begin{array}{c}3703 \\
(81.164)\end{array}$ & & $\begin{array}{l}52001 \\
(7955)\end{array}$ \\
\hline $\mathrm{Lx}$ & & $\begin{array}{c}131789 \\
(296.120)\end{array}$ & & $\begin{array}{c}51425 \\
(5933.49)\end{array}$ \\
\hline
\end{tabular}

Table 3: Goodness-of-fits statistics for the first data set.

\begin{tabular}{ccccc}
\hline \hline Model & $-\hat{\mathbf{L}}$ & AIC & $W^{*}$ & $A^{*}$ \\
\hline \hline BrXELx & $\mathbf{1 2 7 . 6 5 0}$ & $\mathbf{2 6 3 . 3 0 0}$ & $\mathbf{0 . 0 7 7}$ & $\mathbf{0 . 1 5 9}$ \\
BLx & 138.718 & 285.435 & 1.408 & 0.168 \\
ELx & 141.399 & 288.799 & 1.744 & 0.219 \\
& & & & \\
GLx & 138.404 & 282.809 & 1.367 & 0.162 \\
Lx & 164.990 & 333.977 & 1.398 & 0.167 \\
\hline \hline
\end{tabular}



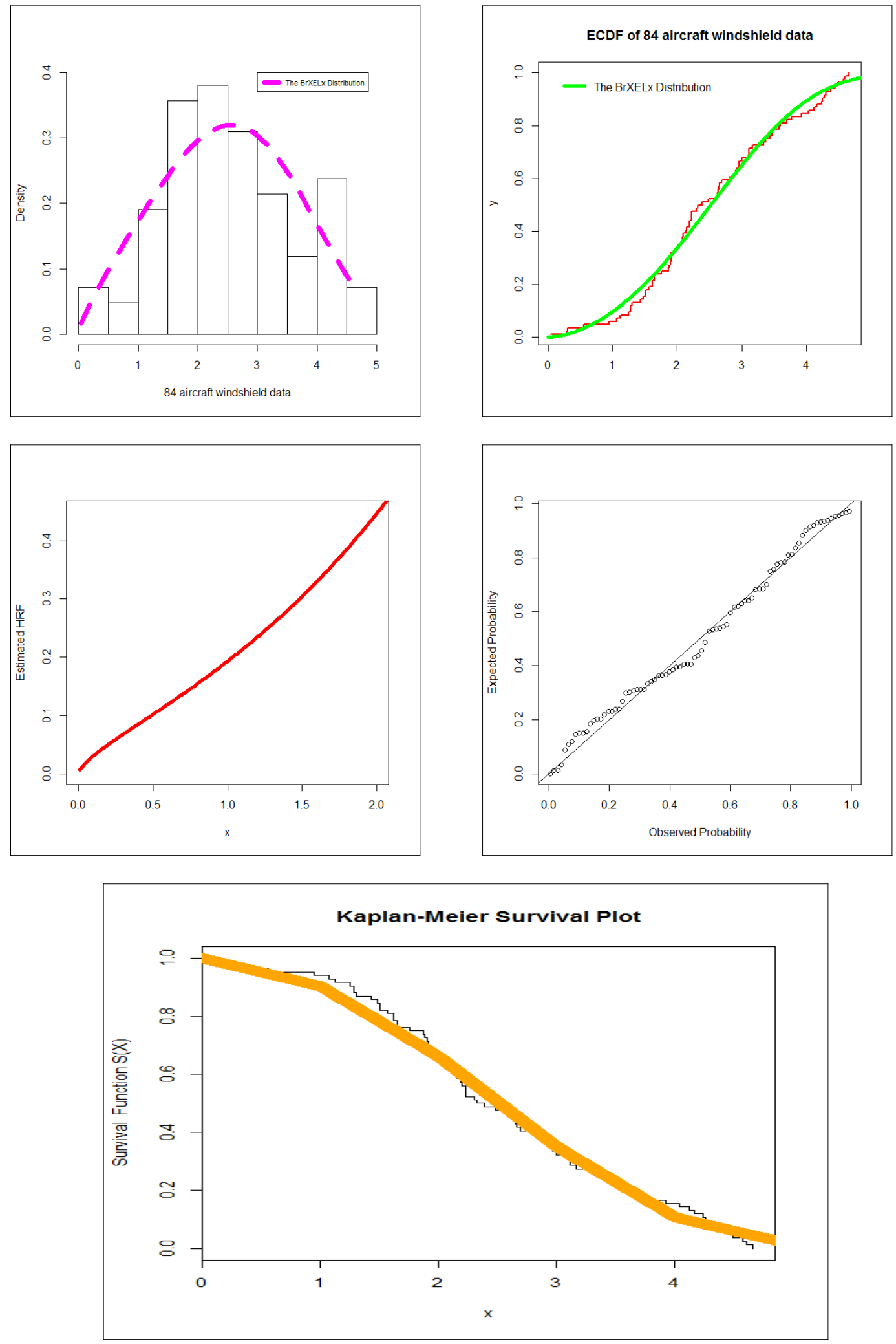

Figure 3: The fitted PDF, CDF, HRF, PP plot and Kaplan-Meier Survival Plot for the first data set. 
Table 4: MLEs and SEs for the second data set.

\begin{tabular}{ccccc}
\hline \hline Model & $\widehat{\alpha}$ & $\widehat{\beta}$ & $\hat{\theta}$ & $\hat{\lambda}$ \\
\hline \hline BrXELx & 1.056 & 11.804 & 0.5414 & 3.154 \\
& $(1.049)$ & $(23.106)$ & $(0.420)$ & $(4.354)$ \\
& & & & \\
BLx & 1.922 & 169.580 & 31.259 & 4.969 \\
& $(0.319)$ & $(339.209)$ & $(316.841)$ & $(50.528)$ \\
& & & & \\
ELx & 1.915 & 32881.9 & & 22971.2 \\
& $(0.348)$ & $(162.223)$ & & $(3209.5)$ \\
& & & & \\
GLx & 1.907 & 39197.6 & & 35842.4 \\
& $(0.321)$ & $(151.65)$ & & $(6945)$ \\
& & & & \\
Lx & & 207019 & & 99269 \\
& & $(301.237)$ & & $(11863.5)$ \\
\hline \hline
\end{tabular}

Table 5: Goodness-of-fits statistics for the second data set.

\begin{tabular}{ccccc}
\hline \hline Model & $-\hat{\mathbf{L}}$ & AIC & $W^{*}$ & $A^{*}$ \\
\hline \hline BrXELx & $\mathbf{9 8 . 2 6 6}$ & $\mathbf{2 0 4 . 5 3 1 7}$ & $\mathbf{0 . 0 4 7 0}$ & $\mathbf{0 . 1 3 0 3}$ \\
BLx & 102.961 & 213.922 & 1.134 & 0.187 \\
& & & & \\
ELx & 103.547 & 213.922 & 1.233 & 0.204 \\
GLx & 102.833 & 211.666 & 1.112 & 0.204 \\
& & & & \\
Lx & 109.299 & 222.598 & 1.127 & 0.186 \\
\hline \hline
\end{tabular}



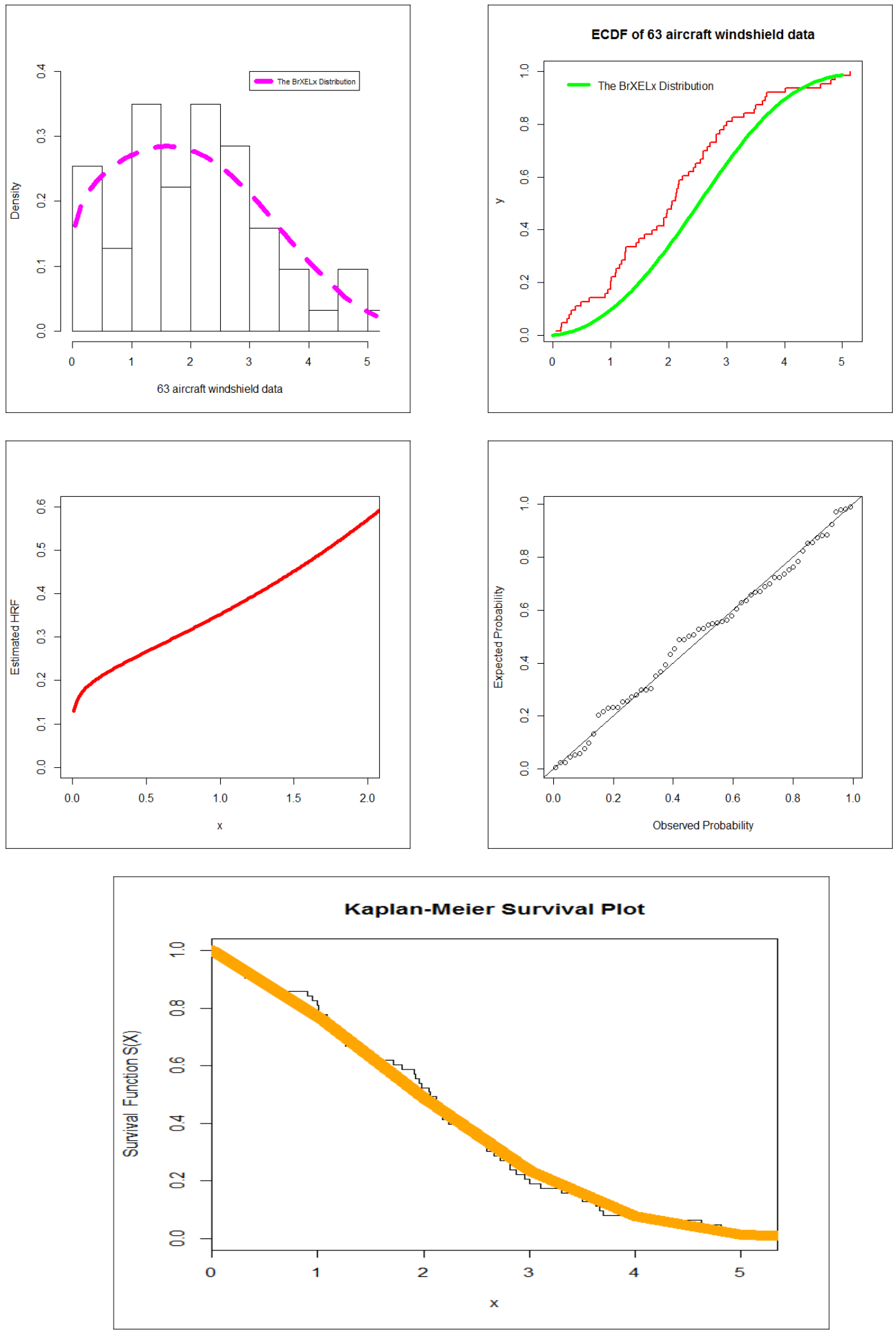

Figure 4: The fitted PDF, CDF, HRF, PP plot and Kaplan-Meier Survival Plot for the second data set. 


\section{Conclusions}

In this article, a new lifetime model called the Burr X exponentiated Lomax (BrXELx) is introduced and studied. The major justification for the practicality of the BrXELx model is based on the wider use of the Lx model. We are also motivated to introduce the BrXELx model since the density of the BrXELx distribution exhibits various important shapes such as the unimodal, the right skewed and the left skewed. The new model can be viewed as a mixture of the exponentiated Lx distribution. It can also be considered as a suitable model for fitting the symmetric, left skewed, right skewed, and unimodal data sets. The maximum likelihood estimation method is used to estimate the BrXELx parameters. We prove empirically the importance and flexibility of the BrXELx in modeling two types of aircraft windshield lifetime data. The proposed BrXELx lifetime model is much better (preferable) than gamma Lomax, beta Lomax, exponentiated Lomax and Lomax models so the exponentiated Lomax, model is a good alternative to these models in modeling aircraft windshield data.

\section{References}

1. Aarset, M. V. (1987). How to identify a bathtub hazard rate. IEEE Transactions on Reliability, 36(1), 106-108.

2. Adel R. S. E., Mobini D. A., Yadollahi F. J. and Azar, A. (2019). A new approach to evaluating entrepreneurial opportunities, Journal of Small Business and Enterprise Development, https://doi.org/10.1108/JSBED-01-2018-0013

3. Afify, A. Z., Nofal, Z. M., Yousof, H. M., El Gebaly, Y. M. and Butt, N. S. (2015). The transmuted Weibull Lomax distribution: properties and application, Pak. J. Stat. Oper. Res., 11(1), 135-152.

4. Atkinson, A.B. and Harrison, A.J. (1978). Distribution of Personal Wealth in Britain (Cambridge University Press, Cambridge).

5. Chen, G. and Balakrishnan, N. (1995). A general purpose approximate goodness-offit test. Journal of Quality Technology, 27, 154-161.

6. Elsayed, H. A. H. and Yousof, H. M. (2019). A new Lomax distribution for modeling survival times and taxes revenue data sets. Journal of Statistics and Applications, forthcoming.

7. Gad, A. M., Hamedani, G. G., Salehabadi, S. M. and Yousof, H. M. (2019). The Burr XII-Burr XII distribution: mathematical properties and characterizations. Pakistan Journal of Statistics, forthcoming.

8. Gupta, R. C., Gupta, P. L. and Gupta, R. D. (1998). Modeling failure time data by Lehman alternatives. Communications in Statistics-Theory and methods, 27(4), 887904.

9. Corbellini, A., Crosato, L., Ganugi, P and Mazzoli, M. (2007). Fitting Pareto II distributions on firm size: Statistical methodology and economic puzzles. Paper presented at the International Conference on Applied Stochastic Models and Data Analysis, Chania, Crete.

10. Cordeiro, G. M., Ortega, E. M. and Popovic, B. V. (2015). The gamma-Lomax distribution. Journal of Statistical computation and Simulation, 85(2), 305-319.

11. Harris, C.M. (1968). The Pareto distribution as a queue service descipline, Operations Research, 16, 307-313.

12. Hassan, A.S. and Al-Ghamdi, A.S. (2009). Optimum step stress accelerated life 
testing for Lomax distibution, Journal of Applied Sciences Research, 5, 2153-2164.

13. Lemonte, A. J. and Cordeiro, G. M. (2013). An extended Lomax distribution. Statistics, 47(4), 800-816.

14. Lomax, K.S. (1954). Business failures: Another example of the analysis of failure data, Journal of the American Statistical Association, 49, 847-852.

15. Murthy, D.N.P. Xie, M. and Jiang, R. (2004). Weibull Models, Wiley.

16. Silva, F. S., Percontini, A., de Brito, E., Ramos, M. W., Venancio, R. and Cordeiro, G. M. (2017). The Odd Lindley-G Family of Distributions. Austrian Journal of Statistics, 46(1), 65-87.

17. Tahir, M. H., Cordeiro, G. M., Mansoor, M. and Zubair, M. (2015). The WeibullLomax distribution: properties and applications. Hacettepe Journal of Mathematics and Statistics, 44(2), 461-480.

18. Yousof, H. M., Afify, A. Z., Hamedani, G. G. and Aryal, G. (2017). The Burr X generator of distributions for lifetime data. Journal of Statistical Theory and Applications, 16, 288-305.

19. Yousof, H. M., Ahsanullah, M. and Khalil, M. G. (2019). A New Zero-Truncated Version of the Poisson Burr XII Distribution: Characterizations and Properties. Journal of Statistical Theory and Applications, 18(1), 1-10.

20. Yousof, H. M., Rasekhi, M., Alizadeh, M. and Hamedani G. G. (2018). The Marshall-Olkin exponentiated generalized $\mathrm{G}$ family of distributions : properties, applications and characterizations. Journal of Nonlinear Sciences and Applications forthcoming. 